\title{
Mobile Primary USER IN Cognitive Radio STATE OF THE ARTS AND RECENT ADVANCES
}

\author{
Amine hamdouchi ${ }^{1}$, Badr Benmammar ${ }^{2}$, Aouatef EI Biari ${ }^{1}$ and \\ Abdellatif Medouri ${ }^{1}$ \\ ${ }^{1}$ National School of Applied Sciences, Tetouan, Morocco \\ ${ }^{2}$ LTT Laboratory, University of Tlemcen, Algeria.
}

\begin{abstract}
The processing of primary user mobility with static or mobile secondary user in the context of cognitive radio (CR) has recently been the subject of several studies and discussions all over the world. These studies are seeking to broaden the horizons of CR implementation beyond the formalism described in the diverse existing standards. The mobility of primary users is likely to reduce the overall performance of the Cognitive Radio Network (CRN) and affects the different phases of the cognitive cycle. Said mobility alters the network's topology, the channel's availability, and affects spectrum sensing. This makes any endeavor aiming to implement CR technology complicated. This paper is devoted to the analysis and discussion of the scientific literature that has addressed the issue of the primary user's mobility.
\end{abstract}

\section{KEYWORDS}

Cognitive radio, Mobility of primary user, Mobility of secondary user, spectrum sensing, channel availability.

\section{INTRODUCTION}

Cognitive radio is considered as a promising approach to solve the current spectrum scarcity and spectrum underutilization problems. It is a fundamental technology enabling manageable, efficient, flexible and reliable spectrum use by adjusting the radio's operating characteristics to the real-time requirements of the ecosystem. The central idea of this new paradigm is that inactive resources or spectrum opportunities can be used by unlicensed users (secondary user) without affecting the performance of licensed users (primary user) [1]. Thus, the spectrum resources will be allocated dynamically rather than statically. Indeed, significant limitations and deficiencies were identified in the last approach thanks to several measurement campaigns which revealed that the spectrum use varies between $15 \%$ and $85 \%$ [2]. In addition, they have shown that the spectrum is exploited sporadically, meaning that frequency bands are used only in particular locations and at particular times [3] as depicted in figure 1. 


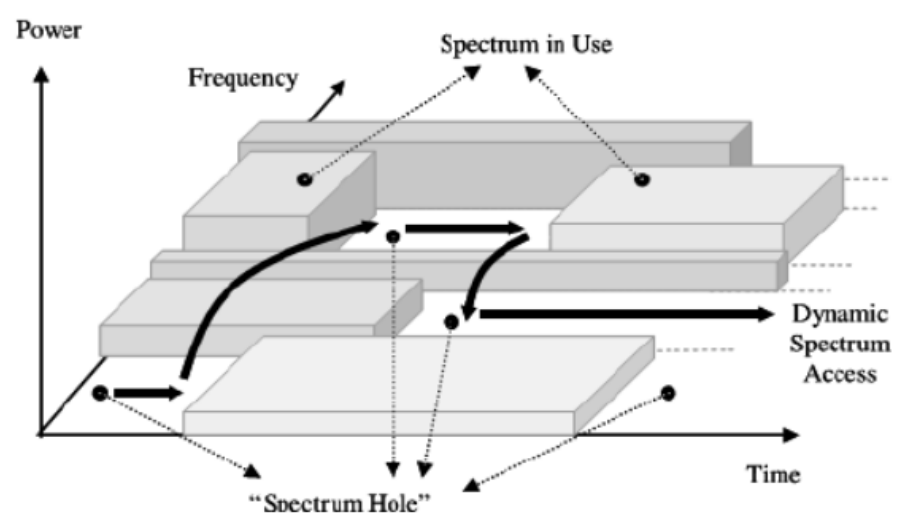

Figure 1. Spectrum hole and mobility concept [4]

The principle of CR relies on understanding the temporal organization and the control states. It follows a series of steps starting with the observation and the orientation of the environment, followed by the creation of the plans and then finally the decision making. As illustrated in figure 2 , the main functions of cognitive radio are: Spectrum sensing, sharing, decision, and mobility [5].

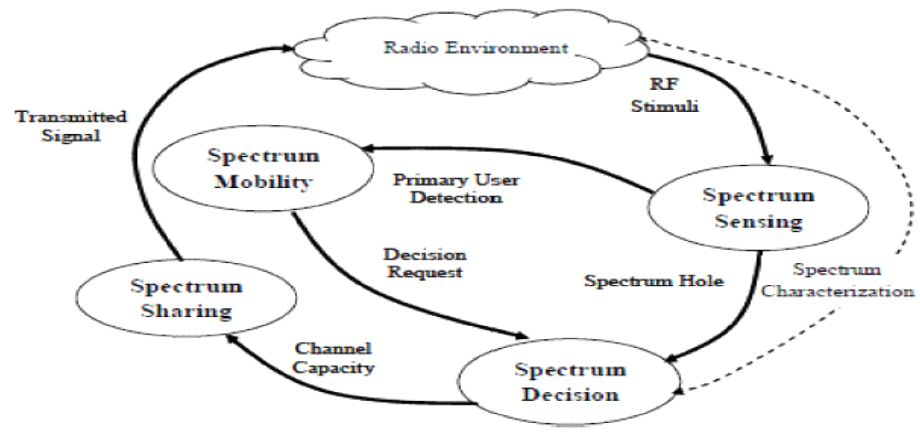

Figure 2. Cognitive Radio Cycle [6]

The IEEE 802.22 Working Group (WG) launched as the first global move to promote a CR-based air-interface (i.e., the PHY and MAC layer specifications) in November 2004 and the standard was published in July 2011 as IEEE 802.22-2011TM. Said Standard is for Cognitive Wireless Regional Area Networks (WRAN) to work in TV Bands [7]

The IEEE 802.22 standard is a CR standard aimed at providing less populated rural areas with broadband access by using vacant television channels. Due to the fact that the levels of industrial noise and ionosphere reflections remain relatively low, the antennas have reasonable dimensions and the propagation characteristics are very good. Television broadcasting in the high VHF and low UHF range are ideal for covering large rural areas with low population density [8]. While the initial version of the standard does not enable mobility, the physical layer of the IEEE 802.22 standard can support mobility of up to $115 \mathrm{~km}$ per hour. Thus, the mobility of the secondary user is supported by this standard, also the ECMA-392 standard support the mobility of secondary users [9].

In this paper, we will not focus on the case where primary users (PUs) are fixed and secondary users (SUs) are mobile because it has been widely discussed in the scientific literature in several 
applications, e.g., CR-MANET in [10,11,12], CR-VANET in [13] or CR-based internet of things [14].

Thus, in this case, the primary network occupies fixed locations and has a deterministic or periodic temporal behavior, making easy the modeling of the cognition cycle for the secondary network in the different phases.

However, the implementation of this technology has posed many challenges, calling into question the effectiveness of the four modules of the cognition cycle: Sensing, spectrum sharing, decision making, and spectrum mobility. Mentioning that they have entered into a bottleneck, new methods are now needed to change this situation. As a result, in [15] the authors have proposed for the first time in 2006 a spectrum prediction mechanism that could improve CR performance as well as the spectrum use. This new mechanism gives the secondary network more intelligence. Thus, its decisions in the sensing phase, decision, and spectrum mobility will be proactive rather than reactive. But for sharing, long-term prediction and multidimensional prediction (time, location and frequencies), several efforts must be made to raise the quality of these phases [16]. Traditional cognitive radio techniques mainly target the primary user and cognitive user in static. For the past few years, the research on the mobility of the user has become a hot research topic, the cognitive network is widely used in multimedia access technology, and the protocols of multimedia access technology are mostly based on the allocation of time. Clearly, one of the main drivers of this awareness is the measurement campaigns that have shown the existence of spectrum opportunities even in cellular networks which stands out for its unrestrained mobility [17]. In practice, the operators deploy network infrastructure to provide advanced service regardless of location, even if the service lasts only a short period of the day. On one hand, these regions (cells) do not reach their maximum use simultaneously and this makes the implementation of CR in cellular networks a very active research topic. On the other hand, the advent of the Internet of Things (IoT) and 5G has reinforced the consideration of primary user mobility. The latter is one of the most significant elements of the wireless system that affects many network characteristic such as network capacity, coverage, and connectivity. It is therefore a fundamental component of any CR implementation in Wireless Next Generation.

Thus, the main objective of this paper is to analyze the case of primary user mobility while highlighting the solutions found as well as the barriers related to this phenomenon. This study distinguishes itself by the fact that it builds on previous work by analyzing an original angle of $\mathrm{CR}$, while the predecessors focused only on one specific technology.

In this paper, we will be concerned with two types of scenario. To facilitate understanding, we appointed the first as "Partial-mobility" and the other as "Global-mobility".

Partial mobility means that only primary user is mobile, while Global mobility means that both types of users are mobile.

In this way, our paper structure will have two main study cases: we will analyze in section 2 the case of partial mobility; we will highlight the progress achieved and emphasize most of the challenges faced by the scientific community. We will end this section by opening a discussion to question the current finding in this scenario. Then in section 3, we will study the case of global mobility, here we will show the low rate of contributions considering the mobility of both PUs and SUs. We then discuss the current literature findings in order to push forward the analyses and to dig into new research ideas, before finally drawing up a general conclusion in section 4 . To the best of our knowledge, this is the first time this topic will be discussed in a paper; the main objective of this work is to highlight current thinking trends and future perspectives of their application. 


\section{Partial Mobility of USers}

\subsection{Impact of Primary user Mobility on Spectrum Sensing}

Spectrum sensing is the key function in implementing cognitive radio, which enables secondary users to identify and utilize vacant spectrum resource allocated to primary users. Traditional techniques, e.g., matched filter (MF), detection energy detector (ED), cyclostationary feature detection, may in practice have different advantages and requirements. Recent new sensing schemes include covariance sensing, probabilistic property-based sensing wavelet analysis, and compressive sensing. It is outstanding that they focus on typical non-mobile CR applications (dynamic access of TV-bands). Nevertheless, none of such methods have taken into account the dynamic position of PU into de sensing process. Lately, research has been made to improve the accuracy and efficiency of these sensing techniques. However, when the PUs are mobile, new problems arise for spectrum sensing despite these efforts.

A key concern in the design of spectrum sensing is the enhancement of the sensing time, i. e. the sensing time that maximizes the transmission rate achievable by SUs, while limiting interference against the PUs. In a mobile network scenario, this issue becomes more challenging than in the traditional case (i.e. the stationary scenario), because in addition to the temporal activity of PU, the spatial effects due to relative mobility between PU and SU must also be considered .In fact, the mobility can profoundly influence the percentage of spectrum opportunities discovered (the spectrum holes that SU can exploit), and then mobility can also affect the transmission throughput that the SU can achieve.

$\operatorname{In}[18]$ the authors have performed an optimization jointly in temporal and spatial domains to account for the PU temporal activity as well as the mobility effects, namely by considering the impact of sensing accuracy on the actual transmission throughput achievable by a SU. More specifically, their theoretical analysis is carried out for an arbitrary network mobility model to prove the existence of a single optimal sensing time that maximizes the mobility-aware sensing Enabled Throughput achievable by a SU.

The mutual distances among the PU and CR users change dynamically with mobility. As result, the capability of the CR users to sense the PU transmissions varies in time. As an example, Fig. 3 shows a CR user that is inside the protection rangel of a mobile PU at a certain time t. After the PU movement, say at time $t+\Delta$, the CR user is out of the PU protection range, thus becoming unable to sense the possible PU transmissions.

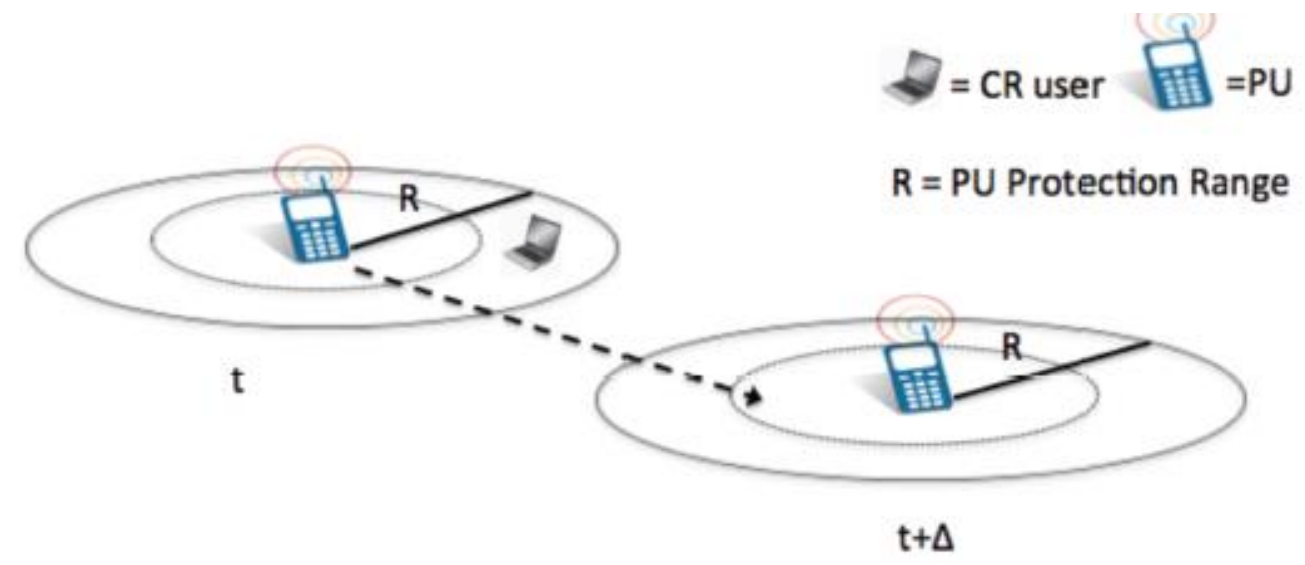

Figure 3. The PU mobility changes the mutual distances between a PU and a CR user [19] 
International Journal of Next-Generation Networks (IJNGN) Vol.12, No.1/2/3, September 2020

In [19] the authors have mentioned that the key issue to deal with the PU mobility consists on the revision of the current design of the spectrum sensing functionality in presence of PU mobility. The spectrum sensing functionality must be able to tune its time parameters, i.e., the sensing time and the transmission time, according to the mobile PU dynamics. To this aim, in their paper, they derived the optimal values for both the parameters in presence of PU mobility. More specifically, regarding the transmission time, they derived an optimal closed-form expression, i.e., the transmission time value that jointly maximizes the spectrum sensing efficiency and satisfies the PU interference constraint.

In [20] firstly, the authors have compared the problem definition of spectrum sensing in static scenario and in partial mobility scenario. Starting from the hypothesis the CR users are assumed to be always in inside the PU protection range (PrR) in static scenario. Therefore, the local sensing for PU signal detection is expressed as a binary hypothesis problem.

Then, they have reformulated the problem of spectrum sensing to fit with the PU mobility with static SU. Finally, they studied the effects of PU with the aim of determining the parameters that affect the spectrum sensing functionality. To that purpose, they introduced two performance measures:

- Detection capability: measures the impact of PU mobility on the probability of detection;

- Mobility-enabled detection capability: a new proposed measure for the expected transmission capability achievable by a SU in the presence of PU mobility.

They found that the detection capacity is affected by five parameters:

- The PU protection range;

- The size of the network;

- The PUs mobility model;

- The spatial distribution of SUs;

- The number of PUs using the same frequency band.

It is also shown in this paper, in the case of PU movement, that the sensing capacity can grow significantly if the PU protection range is smaller than the size of the network area. The uncertainty of spectrum mobility worsens the interference phenomenon. In this case, system capacity analysis becomes a critical issue.

In [21] the authors have depicted to achieve two principals' objectives namely:

- Determine the value of the capacity of the CR users in the network;

- Determine the optimal transmission time of each CR user.

At first, for general PU mobility model, closed-form expressions for the capacity of the CR users and the optimal transmission time of each CR user are derived. Then, these expressions are applied to a widely used model, i.e., the Random Walk mobility model. Finally, the analytical results are verified through simulations.

In order to deal with the challenges appeared with the mobility of PUs, a novel paradigm of sensing has arisen: it is the deep sensing. This new paradigm estimates the time-dependent flatfading gain and primary user's mobile positions jointly at the same time of detecting its emission status. The effectiveness of this approach was deeply investigated [22,23,24]. 
International Journal of Next-Generation Networks (IJNGN) Vol.12, No.1/2/3, September 2020

In [25] the authors have analyzed the effects of multiple mobile PUs on the spectrum sensing to jointly maximize the sensing efficiency and sensing accuracy. To this aim, as first, a new mathematical model (the aggregate PU model) is proposed to effectively describe the cumulative effects of multiple mobile PUs on the spectrum sensing functionality. Then, stemming from this model, closed form expressions for the sensing time and the transmission time that jointly maximize the sensing efficiency and the sensing accuracy are derived.

In [26] the authors have studied the impact of the sensing accuracy on the transmission capacity achievable by the cognitive user $(\mathrm{CU})$ in a mobile primary-user $(\mathrm{PU})$ network scenario.

To this aim, first of all, the spatial-temporal spectrum sensing model is extended to account for the PU mobility effects that influence the number of discovered spatial opportunities. Then, a new performance metric for Cognitive Radio networks, referred to as mobility-aware sensing enabled capacity, is introduced. It measures the actual transmission capacity achievable by the SU in a mobile PU network scenario with a realistic sensing detection capability, namely in the presence of imperfect sensing decisions. The performance assessment highlights the impact of the sensing accuracy on the channel access probability; in particular, it reveals the existence of an optimal sensing time value that maximizes the mobility-aware sensing enabled capacity, which depends on sensing and mobility parameters.

Another way to improve the performance of CR in a mobile scenario is the use of mobility prediction to predict future PU locations according to the pre-collected topology information of PU mobility [27]. In the same frame, a collaborative compressive sensing based approach to estimate both the spectrum power and locations of the PUs is proposed in [28]. The authors have exploited two facts: the relative narrow band nature of the transmitted signals compared with the broad bandwidth of available spectrum and the mobile PUs located sparsely in the operational space. Finally, after exploring many papers and works in this field, we notice that when we are facing PU mobility issues, the proposed solutions generally focus on increasing the level of abstraction often by taking into account two parameters that influence the performance of the sensing in modeling. For example, in deep sensing, localization and sensing are jointly taken into account, or in other cases it is the transmission and sensing times in [20] even in [28] the authors have considered both the power spectrum and location are considered. These problem-solving approaches are specially designed to deal with the complex nature of networks with PU mobility. Thus, for realistic deployment of the CR paradigm in mobile PUs scenarios, the scientific community must not only adapt existing methods or improve them but mainly it has to provide new perceptions, because the existing methods may not support PU mobility. Moreover, how to revise the current design of the spectrum sensing functionality in the presence of PU mobility is still an open question that researchers on CR capacities should consider.

If in static scenarios, the sensing and the transmission times depend mainly on the following three factors:

- The adopted spectrum sensing technique;

- The required interference constraint on the PU transmissions;

- The PU traffic dynamics.

In mobile scenarios, the sensing and the transmission times depend chiefly on the PU mobility.

\subsection{Impact of Primary user on Channel Availability}

The Channel availability is defined as the probability that a licensed channel will be available for unlicensed user communications. Unlike the static scenarios, the channel availability in the 
mobile case varies dynamically over time because of the variations in users' relative positions. Thus, data and information about channel availability allow SU to select the spectrum band with the best communication opportunities.

In most literature, static PUs are assumed where the channel availability does not vary in time. To address this issue, in [29] the authors have designed a strategy to estimate the channel availability based on the relative distances between PUs and SUs. The proposed strategy considers the PU mobility. To develop their model, the authors considered a Random Way Point Mobility (RWPM) for PU, where SUs were considered fixed.

The existence of a communication link between two SUs depends on the transmitting and receiving of the close PUs. To address SU connectivity in CR-MANET, the authors in [30] have analytically characterized this connectivity through continuum percolation and ergodicity theories. Then, they looked into the effect of the primary user' temporal dynamics on the connectivity of the secondary network, this led them to show the incidence of a transition phase phenomenon

The research team distinguished between two cases as follows:

- If PU has some temporal dynamics: the connectivity of the secondary network depends solely on its own density and is independent of the primary traffic.

- If it does not, the connectivity of the secondary network requires putting a density dependent cap on the primary traffic load.

Finally, the authors concluded that the scaling behavior of multi-step delays depends on the instantaneous connection of the secondary network. Notably, they established the scaling law of the minimum multi-hop delay concerning the source-destination distance when the propagation delay is negligible. To develop their model, the authors have considered a fixed secondary network and two mobility models for primary transmitters: the random walk and the random waypoint models.

In [31] a novel mobility-aware Channel-Availability based channel Selection Technique (MCAST) is designed in order to ensure the selection of the channel with the highest channel availability probability in a given temporal interval. To this aim, the channel availability estimation method in the presence of PU mobility is derived firstly. Then, the proposed channel selection techniques efficacy is proved, shown that it takes advantage of the dynamic variation of channel-availability induced by PU mobility. Finally, the effectiveness of MCAST is evaluated in a recently proposed routing metric.

In [32] the authors have showed to design an efficient channel selection strategy, channel availability probability (CAP) and channel quality (CQ) are two key metrics that should be considered. Contrary to static scenario where all the users are immobile, the CAP metric depends only on the primary users' activity whereas the CQ metric remains relatively constant, for mobile scenario the values of both metrics fluctuate not only with time (time-variant) but also over different links between users (link-variant) due to the dynamic variation of primary- and secondary-users' relative positions. In this paper, they have proposed a mobility-aware channel selection technique by jointly accounting for the parameters of time- and link-variant CAP and CQ for MCRNs. Underpinning this novel technique, the contributions of this work include the following:

i) They have derived link-based CAP (L-CAP) estimation based on the relative distances among PUs and CUs in a given temporal interval; ii) They have obtained a mathematical expression that 
International Journal of Next-Generation Networks (IJNGN) Vol.12, No.1/2/3, September 2020

captures estimation of the link based CQ (L-CQ) in mobile scenarios; iii) They have proposed a Link-oriented Channel-Availability and channel-Quality (LCAQ) based channel selection strategy that aims to maximize the link throughput by taking into account two prominent channel selection metrics, namely L-CAP and L-CQ, which are governed by the non-stationary of the network topology induced by the user mobility

\subsection{Conclusion and Discussion of Section 2}

The main concern in the CRN is to identify available free spectrum, this objective will be achieved by sensing the availability of a channel. The issue is that generally most of the studies assume the scenario where both the SU and PU are static. It, therefore, means that the sensing methods developed most commonly are not suitable for the mobile case. In mobile scenario, the issue becomes more challenging than the one associated with the traditional case (i.e.stationary scenario), since in addition to the temporal PU activity also the spatial effects due to the relative mobility between the PUs and CUs have to be taken into account. In fact, the mobility can deeply influence the percentage of discovered spectral opportunities (namely the spectrum holes that the CUs can exploit), and then the mobility can affect also the transmission throughput achievable by the CU.

In addition, a critical challenge for CRN is to design an intelligent channel selection technique. It must choose the best channel by leveraging the dynamic variation of the channel availability driven by PU mobility.

The integration of CR into the mobile scenario implies that either SUs or PUs are constantly exposed to a changing nature, to a variable interference, and high mobility scheduling. This makes cognitive radio nowadays not efficient enough in terms of communication because of the lack of control and optimization adapted to mobility. To remedy this, a support structure adapted to the mobility problems of different types of users should be adopted.

We can summarize the challenges in the case of partial mobility as follows:

- The determination of the spatiotemporal probability of the existence of the spectrum;

- Duration of spectrum sensing;

- Duration of spectrum opportunities;

- $\quad$ Random movements of users;

- $\quad$ Routing problems;

- $\quad$ Energy consumption problems;

- $\quad$ Connectivity problems due to topology change.

What we want to stress in this conclusion is the fact that it is not opportune to use the same approaches to deal with different problems. This is the main stumbling blocks of the realistic deployment of CR in this type of scenario. Thus, we need new perceptions and approaches for this technology in order to take it to a whole new level.

\section{Global Mobility OF USERS}

The In the case of a global mobility of users, the problem of determining SUs links is more complicated because of the movement of both SUs and PUs. Firstly, the communication between the SUs is affected not only by the SUs themselves but also by the activity and the movement of the PUs. The link between neighboring SUs is therefore not stable and the topology of CRN changes frequently. Secondly, opportunistic spectrum access depends on frequent changes in the 
International Journal of Next-Generation Networks (IJNGN) Vol.12, No.1/2/3, September 2020

availability of links for SUs over time and space. Therefore, the topology control and the routing in CRNs show different characteristics compared to traditional wireless networks. In a word, research on dynamic topology control and routing in this type of CRNs is a difficult problem. Thus, research on predicting the temporal availability of links has a significant importance. In this perspective, in [33] the authors sought to present an optimal network topology for routing through a local cognitive topology control algorithm (LCTCA). The LCTCA enables the optimization of routing and the formation of a multi-objective metric of network topology, thanks to combining the availability of links with energy consumption. On this basis, it is designed to select the best network line and diminish the frequency of rerouting; the LCTCA catches the dynamic changes of the topology and then builds a reliable topology under the conditions of network connectivity.

The prediction of an available link must contain the following information:

- The interference of SUs with PUs;

- The rate of spectrum utilization by PUs;

- The mobility of PUs and SUs.

According to the authors, no previous work has considered both energy consumption and link stability to optimize the topology of the CRN. Actually, whenever one of them is considered, the other is ignored. So, the topology of the CRNs cannot reach the optimum.

In [34] the authors have studied the impact of both joint primary-user and secondary user mobility on spectrum sensing in CR networks has been studied. For this reason two performance metrics have been derived analytically: a) the detection capability, which evaluates the impact of the mobility on the SU detection probability; b) the mobility-enabled sensing capacity. Two mobility models were adopted to carry out the theoretical analysis. The authors found out that the sensing capacity increases significantly in the presence of PU mobility if the PU protection range is smaller than the network region size albeit the significant border effect for the RWPconstrained mobility model for PUs. Therefore, in pervasive cognitive radio networks, when unlicensed mobile users are allowed to co- exist alongside mobile licensed users, there is still opportunistic communication expected without much interference. The simulations carried out match the mathematical analysis.

In [35] the authors have built their study on questioning the impact of PUs and SUs mobility on the performance of mobile cellular cognitive radio $(\mathrm{CRCN})$ networks with a real-time traffic. They tried to find answers by deriving the handed-off arrival rate, new call blocking, forced termination, and interruption probabilities of SUs. These metrics were under the assumption that cell dwell time and unencumbered service time are independent exponentially distributed random variables. The obtained numerical results showed that the mobility of PUs and SUs is the key factor and the influencer of the different system performance measures.

In the same vision, in [36] the authors have proposed a new architecture to deal with PU and SU mobility. The suggested architecture will ensure a permanent exchange of information between the primary and secondary networks. Thus, the architecture of the network will be a decisionmaking element, instead of being only a relay of information. The architecture will also reduce spectrum sensing time and increase the efficiency of the detection itself, which is essential to any implementation of CR. Thanks to this the secondary networks will be able to receive the information of the environment in real time and in a permanent manner 
International Journal of Next-Generation Networks (IJNGN) Vol.12, No.1/2/3, September 2020

But some interesting questions have not been addressed in this work to judge its effectiveness. For instance, how the constant exchange of information between networks will affect the network? How often this information should be exchanged for the algorithms to work productively? Supposing that each "network" corresponds to a network cell, then the amount of traffic that needs to be exchanged can be significant, thus influencing the backbone and the overall quality of service. It should also be pointed that the involvement of telecom operators in the development of this kind of algorithms would be the only way to effectively evaluate their effectiveness, otherwise, the work with virtual datasets would not reflect the actual load of cellular networks.

The estimated PU location is subject to many errors due to various reasons, such as; limited observation time, the number of sensors and their coverage, channel variations, and node mobility [37]. Therefore, the assumption of perfect location information makes algorithms that have addressed this issue less practical.

To address this issue, in [38] the authors have proposed a CR power control algorithm that takes into account both uncertainties related to wireless channels and the estimation of the location of PUs that have not been studied in the literature.

Thus, the authors considered the problem of power control in CRNs when the location of the PU and the wireless channel are unknown. They have considered the distance estimation error in the CR-PU links to model the location uncertainties and to derive the channel gain distribution as a function of distance-dependent path loss and shading. Afterwards, they developed an optimization framework for CR power control that maximizes data throughput under the constraint of PU interference power. The simulation results are presented to show that the CR data rate is higher than the existing works.

An evolutionary approach was proposed in [39], where the author has studied the optimal SU packet size in mobile CRNs under fading channels because determining the size of SU packet becomes much more complicated and critical. For this purpose, the PU activity impact and the node mobility impact are studied jointly. The author investigates a CRN where both PUs and SUs are mobile. He considered the Random Waypoint as the mobility model for both types of user. He found that the PU activity and the mobility of both SUs and PUs have significant impacts on the SU packet size.

\subsection{Conclusion and Discussion of Section 3}

Our investigation in the scientific literature has shown that studies on the mobility of both PU and SU are rare and, moreover, scattered. Most efforts to find solutions to mobility simply indicate or ask questions about the scenario where PU and SU are both mobile, without touching the heart of the subject. We envision that this scenario of mobility will emerge as a major challenge for the future of $\mathrm{CR}$, if the characterization of the radio environment involves the estimation of the following key elements [40]:

- Channel Identification ;

- Channel Capacity Estimation ;

- Channel Switching Delay ;

- Channel Interference Estimation ;

- Channel Holding Time ;

- Channel Error Rate ;

- Subscriber Location ;

- Path Loss. 
International Journal of Next-Generation Networks (IJNGN) Vol.12, No.1/2/3, September 2020

Accordingly, these elements should be revised with the incorporation of the mobility of both types of users in the context of CR. Since this scheme of the characterization of the radio environment assumes that both users are static, whereas new problems will arise that were not included in this traditional approach. Thus, this mobility requires rethinking not only the main features characterizing the radio environment but the whole concept of CR.

To deal with sensing problems, new perceptions and strategies are needed to get the CR out of the bottleneck. This case implies that cooperation should not be restricted only between SU, but should be extended to include PU. Therefore, other questions will arise for the first time in the context of the CR, for example:

- How SU and PU cooperate in cooperative sensing?

- How to select the cooperating SU and PU?

- How to perform cooperative sensing in a stable and reliable manner?

Another proposition is to take advantage of the explosion of extensive geo-localized data related to the movement of different types of terminals. This will make it possible to quantitatively study individual and collective mobility models, and to generate models capable of capturing and reproducing spatiotemporal structures. This will also contribute to the deployment of spectrum maps that are able to provide more accurate information.

We also think that it is important to incorporate online machine learning algorithms in the different phases of the cognitive cycle to take advantage of its opportunities compared to batch learning algorithms [41].

Mobile scenarios are totally different from traditional static ones, hence, the need to innovate and produce other mathematical methods and models that can take this complexity into account. Multi-objective optimization can also be an effective mean of studying dynamic access to spectrum, since the latter involves several variables that require joint processing, hence the need to find a compromise between them.

Also, the issue of the suitability of PU mobility models has recently become a source of criticism. In [42] the authors have argued that realistic mobility models are important for understanding the performance of routing protocols in the cognitive Internet (CI) (same for CR), especially when the behaviours and activities of the PUs are taken into account. Currently, the primary user's mobility models are generally simplistic spectrum handover models or traditional mobility models that do not reflect realistic spatiotemporal characteristics. Therefore, the effect of PU mobility should be closely integrated with the available spectrum time and the appearance behaviour of the PUs. A new PU mobility model called TSS (temporal mobility, spatial mobility, and spectrum mobility) was proposed in [42]. In the same vision, in [43] the authors have noted that the effect of PU mobility has not received enough attention in the CRCN because it is a recent research topic. Thus, the service interruption of SUs due to the arrival of PU and its mobility must be taken into account for the $\mathrm{CRCN}$ performance analysis.

Even in [44] the authors have mentioned that although previous studies have raised questions about the conventional cellular network and CR networks, they have generally focused on single subject research topics such as priority scheduling in conventional cellular networks, or the intercellular activity of SUs in CR networks. But the problem lies in the joint consideration of these research subjects. In the model proposed by these authors, the cognitive base station (CBS) treats a number of mobile stations (MS) as secondary mobile stations (SMS), while others are considered primary mobile stations (PMS). In this overlay approach: CBSs share the same radio 
International Journal of Next-Generation Networks (IJNGN) Vol.12, No.1/2/3, September 2020

channels to communicate simultaneously between SMS and PMS pairs. The classification of an MS as a PMS or SMS depends on its location in a macro cell. (This is a new way of classifying SU and PU).Thus, each cell is divided into three zones: A) opportunistic or cognitive, B) protected and regular, or C) primary. In an inner area A, the terminal behaves as a secondary receiver (SMS) while inside area $\mathrm{B}$ or $\mathrm{C}$ it behaves as a normal mobile receiver, while an MS in area $\mathrm{C}$ is considered only as PMS. Protected area $\mathrm{B}$ attenuates the signal transmitted to SMS below the acceptable signal level received at the PMS in area $\mathrm{C}$. The development and innovation of other classification methods such as the one described in this article can reduce the complexity of CR implementation in the mobile case.

\section{Conclusions}

Our analysis of the existing literature on CR and mobility has shown that user mobility within the context of CR is a subject of deep and crucial consideration for good spectrum management. We can say that in reality, there is no shortage of spectrum, but only the lack of advanced techniques for perfect spectrum use. This paper presented a new taxonomy of work based on the mobility of primary users. The main objective of our work was to highlight this phenomenon within the context of CR. In fact, it is a recent research topic that doesn't reach its maturity yet to enables CR to become the foundation of the "Next Generation Network." This work can help new researches to focus on the mobility of PUs. We believe that the limits of the CR in telecommunications have not yet been reached and that many efforts are still needed, as the future would be a world of trillions of objects such as IoT ones, requiring continuous spectrum functionality. Traditional communication technologies would not withstand this situation. This means the development of techniques that support the mobility of different types of users has become an obligation

\section{REFERENCES}

[1] Mitola, J., \& Maguire, G. Q. (1999). Cognitive radio: making software radios . more personal. IEEE personal communications, 6(4), pp.13-18.

[2] Federal Communications Commission), (2003) "Spectrum Policy Task Force Report FCC 03-289," ET Docket No. 03-237, no. Nov., pp. 1-31

[3] Chiang, R. I., Rowe, G. B., \& Sowerby, K. W. (2007, April). A quantitative analysis of spectral occupancy measurements for cognitive radio. In 2007 IEEE 65th Vehicular Technology ConferenceVTC2007-Spring (pp. 3016-3020). IEEE.

[4] I. F. Akyildiz, W. Y. Lee, M. C. Vuran and S. Mohanty, (2006), "NeXt Generation / Dynamic Spectrum Access / Cognitive Radio Wireless Networks: A Survey", Computer Networks, pp. 21272159

[5] Haykin, S. (2005). Cognitive radio: brain-empowered wireless communications. IEEE journal on selected areas in communications, 23(2), pp.201-220

[6] I. F. Akyildiz, W.-Y. Lee, K. R. Chowdhury (2009): "CRAHNs: Cognitive Radio Ad Hoc Networks", Ad Hoc Networks, Elsevier, Vol. 7, No. 5, July 2009, pp. 810-836

[7] IEEE Working Group on Wireless Regional Area Networks, "Enabling rural broadband wireless access using cognitive radio technology in TV whitespaces," http://www.ieee802.org/22/ (Last accessed Mar. 2015)

[8] Benmammar, B., \& Amraoui, A. (2013). Radio resource allocation and dynamic spectrum access. ISTE.

[9] Khattab, A., \& Bayoumi, M. A. (2015). Standardization of cognitive radio networking: a comprehensive survey. annals of telecommunications-annales des télécommunications, 70(11-12), pp.465-477.

[10] Jabbar, W. A., Ismail, M., Nordin, R., \& Arif, S. (2017). Power-efficient routing schemes for MANETs: a survey and open issues. Wireless Networks, 23(6), pp. 1917-1952 
International Journal of Next-Generation Networks (IJNGN) Vol.12, No.1/2/3, September 2020

[11] Singh, K., \& Moh, S. (2016). Routing protocols in cognitive radio ad hoc networks: A comprehensive review. Journal of Network and Computer Applications, 72, pp.28- 37.

[12] Soni, S., \& Srivastava, S. (2016). Survey of Quality of Service Routing Protocol in MANET. International Journal of Computer Applications, 136(13).

[13] Singh, K. D., Rawat, P., \& Bonnin, J. M. (2014). Cognitive radio for vehicular ad hoc networks (CRVANETs): approaches and challenges. EURASIP journal on wireless communications and networking, 2014(1), 49

[14] Khan, A. A., Rehmani, M. H., \& Rachedi, A. (2017). Cognitive-radio-based internet of things: Applications, architectures, spectrum related functionalities, and future research directions. IEEE wireless communications, 24(3), pp.17-25.

[15] Kumar Acharya, P. A., Singh, S., \& Zheng, H. (2006, August). Reliable open spectrum communications through proactive spectrum access. In Proceedings of the first international workshop on Technology and policy for accessing spectrum (pp. 5-es).

[16] Wu, J., \& Li, Y. (2017, April). A survey of spectrum prediction methods in cognitive radio networks. In AIP Conference Proceedings (Vol. 1834, No. 1, p. 020018). AIP Publishing LLC.

[17] Carolan, Emmett, Seamus C. McLoone, and Ronan Farrell ( 2015) "Predictive modelling of cellular load." Signals and Systems Conference (ISSC), 2015 26th Irish.IEEE, 2015

[18] Marino, F., Paura, L., \& Savoia, R. (2015). On spectrum sensing optimal design in spatial-temporal domain for cognitive radio networks. IEEE Transactions on Vehicular Technology, 65(10),pp. 84968510 .

[19] Cacciapuoti, A. S., Akyildiz, I. F., \& Paura, L. (2013). Optimal primary-user mobility aware spectrum sensing design for cognitive radio networks. IEEE Journal on Selected Areas in Communications, 31(11),pp. 2161-2172.

[20] Cacciapuoti, A. S., Akyildiz, I. F., \& Paura, L. (2011, September). Primary-user mobility impact on spectrum sensing in cognitive radio networks. In 2011 IEEE 22nd International Symposium on Personal, Indoor and Mobile Radio Communications (pp. 451-456). IEEE.

[21] Jia, M., Zhang, G., \& Gu, X. (2015, October). Optimal primary-user mobility aware parameters design of spectrum sensing in cognitive radio networks. In 2015 International Conference on Information and Communication Technology Convergence (ICTC) (pp. 1237-1239). IEEE.

[22] Li, B., Li, S., Nallanathan, A., \& Zhao, C. (2015). Deep sensing for future spectrum and location awareness 5G communications. IEEE Journal on Selected Areas in Communications, 33(7), pp.13311344

[23] Li, B., Nan, Y., Zhao, C., \& Nallanathan, A. (2015, July). Deep sensing for future 5G communications with mobile primary users. In 2015 IEEE International Conference on Digital Signal Processing (DSP) (pp. 521-525). IEEE

[24] Li, B., Hou, J., Li, X., Nan, Y., Nallanathan, A., \& Zhao, C. (2016). Deep sensing for space-time doubly selective channels: when a primary user is mobile and the channel is flat rayleigh fading. IEEE Transactions on Signal Processing, 64(13), pp.3362-3375.

[25] Cacciapuoti, A. S., \& Caleffi, M. (2015). Spectrum sensing in small-scale networks: Dealing with multiple mobile PUs. Ad Hoc Networks, 33, pp.209-220

[26] Paura, L., \& Savoia, R. (2013, October). Mobility-aware sensing enabled capacity in Cognitive Radio networks. In 2013 IEEE International Workshop on Measurements \& Networking (M\&N) (pp. 179183). IEEE.

[27] Bütün, İ., Talay, A. Ç., Altilar, D. T., Khalid, M., \& Sankar, R. (2010, April). Impact of mobility prediction on the performance of cognitive radio networks. In 2010 Wireless Telecommunications Symposium (WTS) (pp. 1-5). IEEE

[28] Liu, L., Han, Z., Wu, Z., \& Qian, L. (2011, December). Collaborative compressive sensing based dynamic spectrum sensing and mobile primary user localization in cognitive radio networks. In 2011 IEEE Global Telecommunications Conference-GLOBECOM 2011 (pp. 1-5). IEEE

[29] Cacciapuoti, A. S., Caleffi, M., Paura, L., \& Rahman, M. A. (2015). Channel availability for mobile cognitive radio networks. Journal of Network and Computer Applications, 47, pp.131-136.

[30] Ren, W., Zhao, Q., \& Swami, A. (2013). Temporal traffic dynamics improve the connectivity of ad hoc cognitive radio networks. IEEE/ACM Transactions on Networking,22 (1),pp.124-136.

[31] Rahman, M. A., Savoia, R., \& Uddin, M. M. (2015). MCAST: mobility-aware channel-availability based channel selection technique. ICNS 2015, 93. 
International Journal of Next-Generation Networks (IJNGN) Vol.12, No.1/2/3, September 2020

[32] Rahman, M. A., Asyhari, A. T., Bhuiyan, M. Z. A., Salih, Q. M., \& Zamli, K. Z. B. (2018). L-CAQ: Joint link-oriented channel-availability and channel-quality based channel selection for mobile cognitive radio networks. Journal of Network and Computer Applications, 113,pp. 26-35

[33] Liu, S., Wang, Y., \& Cui, C. (2017). A Topology Control and Routing Method in MCRNs Based on Power Consumption and Link Stability. Wireless Personal Communications, 92(4), pp.1347-1363

[34] Okell, K., El-Malek, A. H. A., Elsabrouty, M., \& Abo-Zahhad, M. (2019, April). Spectrum Sensing Performance Analysis for Mobile Primary and Secondary Users in Cognitive Radio Networks. In 2019 Wireless Days (WD) (pp. 1-4). IEEE

[35] Serrano-Chavez, J. O. S. E., Hernandez-Valdez, G. E. N. A. R. O., Cruz-Pérez, F. A., CastellanosLopez, S. L., Andrade-Gonzalez, E. A., Reyes-Ayala, M. A. R. I. O., \& Miranda-Tello, J. R. (2015). Impact of mobility on the performance of cognitive radio mobile cellular networks with real-time traffic. In Proc. DNCOCO'15 (pp. 236-242).

[36] Hamdouchi, A., El Biari, A., Benmammar, B., \& Tabii, Y. (2017, November). Towards the Use of Cognitive Radio to Solve Cellular Network Challenges. In Interactive Mobile Communication, Technologies and Learning (pp. 3-10). Springer, Cham.

[37] Wang, J., Chen, J., \& Cabric, D. (2013). Cramer-Rao bounds for joint RSS/DoA-based primary-user localization in cognitive radio networks. IEEE Transactions on Wireless Communications, 12(3), pp. 1363-1375

[38] Wang, J., Chen, J., Lu, Y., Gerla, M., \& Cabric, D. (2014). Robust power control under location and channel uncertainty in cognitive radio networks. IEEE Wireless Communications Letters, 4(2), pp.113-116

[39] Song, Y. (2015, April). Optimal secondary user packet size in mobile cognitive radio networks under fading channels. In 2015 IEEE Conference on Computer Communications (INFOCOM) (pp. 163171). IEEE.

[40] Masonta, M.T., Mzyece, M. and Ntlatlapa, N. (2013) 'Spectrum decision in cognitive radio networks: A survey.' IEEE Communications Surveys \& Tutorials, Vo.15 No.3, pp.1088-1107

[41] Fontenla-Romero, Ó., Guijarro-Berdiñas, B., Martinez-Rego, D., Pérez- $\mu$ Sánchez, B., \& PeteiroBarral, D. (2013). Online machine learning. In Efficiency and Scalability Methods for Computational Intellect (pp. 27-54). IGI Global.

[42] Zhang, Neng, et al (2013). "TSS: A Primary User Mobility Model in Cognitive Internet."Innovative Mobile and Internet Services in Ubiquitous Computing (IMIS), Seventh International Conference on. IEEE, 2013

[43] Corral-Ruiz, A. L. E., Cruz-Perez, F. A., Castellanos-Lopez, S. L., Hernandez- Valdez, G., \& RiveroAngeles, M. E. (2017). Modeling and performance analysis for mobile cognitive radio cellular networks. EURASIP Journal on Wireless Communications and Networking, 2017(1), 159.

[44] Alam, A. S., Dooley, L. S., \& Poulton, A. S. (2011, June). Dynamic spectrum access based on cognitive radio within cellular networks. In 2011 Wireless Advanced (pp. 85-89). IEEE. 